\title{
Second messengers in endocrinology: a mini-review of the cyclic nucleotides
}

\begin{abstract}
In this mini-review the developments in the cyclic nucleotide "second messenger" system are discussed. Since the discovery in 1957 knowledge about the downstream effects of the cyclic nucleotides has increased enormously. The EPAC system and the newly discovered cyclic-di-monophosphate system will have promising therapeutic applications in the future and are new challenges.
\end{abstract}

Keywords: Second messenger, Cyclic nucleotides, EPAC system, Cyclic-dimonophosphate, cAMP, Cyclic nucleotide phosphodiesterases
Volume 5 Issue 6 - 2017

\section{Michael AB Naafs}

Dutch Internist Endocrinologist, Naafs International Health Consultancy, Netherlands

Correspondence: Michael AB Naafs, Dutch Internist Endocrinologist, Health Consultant at Naafs International Health Consultancy, Rhodoslaan 20,7577KN, Oldenzaal,The Netherlands, Tel +3I68I589079,

Email michael.naafs@hotmail.com
Abbreviations: PKA, protein kinase; BDNF, brain derived neurotrophic factor; CRE, cAMP response element; ROS, reactive oxygen species; PKG, protein kinase G; PTH, parathyreoid hormone; NEP: neural endopeptidase

\section{Introduction}

Second messengers are molecules inside cells that act to transmit signals from a receptor to a target. The term second messenger was coined upon the discovery of these substances in order to distinguish them from hormones and other molecules that function outside the cell as "first messengers" in the transmission of biological information. ${ }^{1}$

Many second messengers are small and therefore diffuse rapidly through the cytoplasma enabling information to move quickly throughout the cell. As elements of signalling pathways second messenger scan serve to integrate information when multiple independent upstream inputs influence the rates of synthesis and degradation of the second messenger. In addition second messengers can have multiple downstream targets thereby expanding the scope of signal transmission.

A large number of second messengers have been characterized including cyclic nucleotides, hydrophylic molecules eg cyclic adenosine monophosphate or cAMP and cyclic guanosine monophosphate or cGMP (hydrophobic molecules), ions (eg $\mathrm{Ca}^{2+}$ ), phospholipid derived molecules (eg., inositol triphosphate, phosphatidyl inositol ) and even gasses as nitric oxide ( $\mathrm{NO}), \mathrm{H}_{2} \mathrm{~S}$ and carbon monoxide (CO).

The calcium ion $\mathrm{Ca}^{2+}$, which can diffuse both through cytosol and across cellular membranes, has a critical role in the rapid response of neurons and muscle cells. At rest cells maintain a low concentration of $\mathrm{Ca}^{2+}$, in the cytoplasma expending energy to pump these ions out of the cell. When activated neurons and muscle cells increase their cytoplasmic $\mathrm{Ca}^{2+}$, concentrations by opening channels in the cell membrane which allow $\mathrm{Ca}^{2+}$, ions outside the cell to enter rapidly. For the purpose of this mini-review only the cyclic nucleotides are discussed.

\section{The cyclic nucleotides}

The cyclic nucleotide cAMP is synthesized by adenylate cyclase enzymes which are downstream of G-proteins (guanine nucleotide binding proteins) and receptors. ${ }^{2}$ Cyclic AMP generated by adenyl cyclase has three major targets in most cells.

1. The cyclic AMP-dependent protein kinase (PKA)

2. cAMP regulated guanine nucleotide exchange factors termed EPACs (exchange protein directly activated by cAMP)

3. Via PKA phosphorylation by a cAMP response binding protein called CREB. ${ }^{4-6}$

The most common downstream effector of CAMP is protein kinase A (PKA). PKA is normally inactive as a tetrameric holoenzyme (two catalytic and two regulatory units). The regulatory unit always blocks the catalytic center of the catalytic unit. The regulatory unit dissociates from the catalytic subunit. The free catalytic subunit interacts with proteins to phosphorylate Ser or The residues, thus providing a cellular response.

PKA can phosphorylate a diverse array of physiological targets such as metabolic enzymes and transport proteins and numerous regulatory proteins including other protein kinases, regulation of glycogen, sugar and lipid metabolism, ion channels and transcription factors. ${ }^{3-6}$ Cellular transcription factors as the neutrophin BDNF (Brain Derived Neurotrophic Factor), tyrosine hydroxylase and many neuropeptides bind to certain DNA sequences called cAMP response elements (CRE) thereby increasing or decreasing the transcription of the downstream genes.?

Protein synthesis by PKA directly activates CREB which bind the cAMP response element (CRE). The activated CREB protein then binds to a CRE region and is then bound to CBP (CREB binding protein) which co activates it allowing it to swith within genes on and off and altering the transcription.

CREB proteins have many functions in many different organs. CREB proteins in neurons are thought to be involved in short-and 
long-term memory. ${ }^{8}$ CREB proteins also have an inhibiting effect on T-cell proliferation and its? Effector effects via CAMP. ${ }^{9}$

To integrate extracellular signals from membrane receptors with diverse intracellular signalling pathways the system uses cAMPregulated guanine nucleotide exchange factors (GEFs). For that purpose small GTPases operate as binary switches that exist in GTP or in GDP ligand formation. ${ }^{10}$ They are involved in processes like cell adhesion, phagocytosis, apoptosis and gene expression. ${ }^{11}$

The two GEFs regulated by cAMP are able to activate members of the Ras small GTPase family Rap1 and Rap2.

(EPAC1 and EPAC2) New paragraph? The EPAC pathway is involved in immune, lung and neuronal function.EPAC1 regulates the differentiation of monocytes to macrophages and subsequently controls cellular phagocytosis as well as the production of inflammatory mediators and apoptosis in human leukocytes. ${ }^{12}$ EPAC1 modulates integrin function most probably by barrier function and leukocyte adhesion and transmigration. ${ }^{13}$

Mature lymphocytes with their antigen-specific receptors are in a permanent circulation from the blood stream to the lymphoid organs and represent the main mediators of the adaptive immune response. In $\mathrm{B}$-cells signalling by the antigen-specific $\mathrm{B}$-cell antigen- receptor complex (BCR) is a central step to maintain homeostasis and proper immune function with cAMP in the role of a central mediator. ${ }^{14}$ Expression of EPAC1 has been reported in B cell chronic lymphocytic leukemia. ${ }^{15}$

EPACS represent a novel key effector in the lung due to their capacity to modulate airway inflammation and proliferation and as part of the signalling cascade triggered by beta 2-adrenocepter agonists that target cAMP to alleviate the symptoms of asthma. ${ }^{16}$

It has been postulated that the developmental regulation of EPAC expression promotes axon growth and regeneration in the nervous system. ${ }^{17}$ Epac2 signalling inhibition induced early afterdepolarization in ventricular myocytes in the heart. The underlying mechanism involves an increase in mitochondrial reactive oxygen species (ROS) and activation of the late sodium current (i Nalate)? Inhibition of EPAC2 caused venticular tachycardia, torsades de pointes and sudden death. ${ }^{18}$

In the pancreas the production of the incretin hormone glucagonlike-peptide-1 (GLP-1) is stimulated by activation of PKA and EPAC2. ${ }^{19}$ In the brain EPAC2 may regulate synaptic plasticity and thus control higher brain functions such as memory and learning. ${ }^{20,21}$

Pharmacological manipulation of cAMP levels exerts beneficial effects through the regulation of EPAC. For example EPAC2 selective agonists could promote insulin secretion from pancreatic beta cells whereas EPAC1 selective agonists may be useful in the treatment of vascular inflammation. In contrast EPAC1 and EPAC2 antagonists could both be useful in the treatment of heart failure. ${ }^{22}$

Cyclic nucleotide phosphodiesterases form another family of important signalling proteins. Their activities are regulated via the rate of gene transcription as well as by second messengers (cyclic nucleotides or $\mathrm{Ca}^{2+}$ ) and interactions with other signalling proteins such as beta-arrestin and proteinkinases. PDEs hydrolyze the cyclic 3,5-phosphodiester bound in cAMP and cGMP thereby terminating their action, Phosphodiesterase 4 (PDE4) is the predominant degrading enzyme expressed in inflammatory cells. Selective PDE4 inhibitors can play a therapeutic role in various inflammatory diseases as asthma, psoriasis, inflammatory bowel disease etc. ${ }^{23,24}$

Cyclic AMP promotes the release of the anti-inflammatory mediators (eg IL-10) by immune cells. A decrease in PDE 4 increases a cAMP level which leads to increased transcription of genes that have CRE sites, including the gene for IL-10. PDEs are drug targets for the treatment of diseases as asthma, COPD, cardiovascular diseases as heart failure, atherosclerotic coronary and peripheral arterial disease and neurological disorders. ${ }^{24-26}$ Recently cyclic di-adenosine monophosphate was discovered (c-di-AMP). It is a broadly conserved messenger for bacterial growth and infection. However the molecular mechanisms of c-di-AMP signalling are still poorly understood..$^{27,28}$

\section{Cyclic GMP}

The signalling pathways that regulate the synthesis of cyclic GMP in cells include hormonal regulation of transmembrane guanylate cyclases such as via the natriuretic peptide receptor (ANP) and the activation of soluble guanylate cyclases by nitric oxide (NO). Unlike cAMP the second messenger cGMP has established signalling roles in only a few cell types. The synthesis is regulated by NO or by the natriuretic peptide hormones ANP, BNP (brain natriuretic peptide) and CNP (C-type natriuretic peptide). ${ }^{29} \mathrm{NO}$ is synthetisized from arginine and oxygen by the NO synthetase. It diffuses freely through the plasma membrane of the cells. It activates soluble guanyl cyclases to produce cGMP. NO synthesis by endothelial cells is stimulated by the neurotransmitter acetylcholine. The function of NO is blood vessel dilatation and smooth muscle relaxation.

Nitric Oxide Synthetase (NOS) exists in 4 isoforms: the neuronal type 1 isoform (nNOS), the inducible type 2 isoform (iNOS), the endothelial type 3 isoform (eNOS) and the mitochondrial isoform $(\mathrm{mNOS}) .{ }^{30}$ eNOS is expressed in endothelium, cardiac myocytes, renal mesangial cells, osteoblasts and platelets and is involved with vasodilation (30) nNOS serves nerves and skeletal muscles and performs a role in cell communication and plays also a role in regulating blood pressure in healthy humans. ${ }^{31}$ iNOS is expressed in macrophages, Kupffer cells, neutrophils, fibroblasts and vascular smooth muscle cells and endothelium. It is involved in the immune defense against pathogens. ${ }^{32}$ mNOS regulates ATP synthesis in the mitochondrial matrix. ${ }^{33}$

The transmembrane receptors for ANP, BNP and CNP are ligandactivated guanyl cyclases. Phosphorylation of serine residues in the kinase domain is important for activity. Dephosphorylation of these residues leads to desensitation of the receptor. After phosphorylation of the serine residues guanyl cyclase production is stimulated. Downstream reactions of cGMP are the activation of protein kinase $\mathrm{G}(\mathrm{PKG})$, gate keeping the ion channels and the formation of a cGMP modulated phosphodiesterase. Pharmacological important effects of elevated cGMP levels include modulation of platelet activation and relaxation of smooth muscle. PDEs hydrolyze the cyclic 3-5phosphodiester bond in CAMP and cGMP thereby terminating their action. $^{34,35}$

While the natriuretic peptides use the guanyl cyclase system hormones like glucagon, epinephrine and parathyreoid hormone (PTH) use the adenylate cyclase system. States of excess or low PTH production can be studied by measuring nephrogenous cAMP excretion and phosphaturia (TmPO4/GFR). States of end-organ resistance to $\mathrm{PTH}$ can be studied also in this way. ${ }^{36,37}$ 
The peptide hormones use the hormone receptor complex. Pharmacologic drugs use the ligand-receptor complex which is very similar to the hormone-receptor complex. However in this case the drug is the ligand. ${ }^{38}$

\section{Selected drugs that stimulate cAMP formation are}

a. Salmeterol, a beta2agonist used in asthma and COPD.

b. Haloperidol, a D2, D3, D4 antagonist used in schizophrenia.

i. Metoclopramide, a D2, 5 HT4 antagonists used in nausea and vomiting.

ii. Desmopressin, a V2 receptor agonist used in diabetes insipid us.

c. Drugs that decrease cAMP formation are for example:

d. Metprolol, a beta 1 antagonist used in angina pectoris, hypertension and heart failure.

e. Morphine, an u agonist? Used in pain relief.

f. Ibuprofen, a non-selective COX-inhibitor used in inflammation or pain.

g. Carbagoline, a D2 agonist used in Parkinson's disease.

h. Drugs that use the NO-cGMP system are for example:

i. Nitroglycerin, isosorbide mononitrate. They are NO donors used in angina pectoris

j. Sodium nitroprusside used in hypertensive emergencies is a NO donor.

k. Niorandil, a $\mathrm{K}+$ channel opener and $\mathrm{NO}$ donor used in angina pectoris

1. Nifendipine, a NO releaser used in angina pectoris and hypertension.

\section{Synthetic natriuretic peptides are}

i. Nesritide, a synthetic BNP that promotes vasodilatation, diuresis, natriuresis and used in heart failure.

ii. Ecadotril-Neural Endopeptidase (NEP), catalyses BNP degradation, used in CHF.

\section{PDE inhibitors}

a. They act by modulating the levels of second messengers.

b. PDE 3 inhibitors, Amrinone, Milrinone used in $\mathrm{CHF}$ as vasodilators.

c. PDE 5 inhibitors, Sildanafil, Tadalafil used in erectile dysfunction. Produce vasodilatation by NO dependent elevation of cGMP in vascular smooth muscle.

d. Theophylline, Aminophylline, Non-selective PDE inhibitors used in asthma and COPD.

e. Pentoxyphylline, a PDE 3 inhibitor with rheologic modifying properties. Improves the microcirculation.

f. Cilostazol, PDE 3 inhibitor that promotes vasodilatation and inhibits platelet aggregation. g. Dipyridamole, a PDE 5 inhibitor. Inhibits thrombocytes aggregation. Prevents uptake and degranulation of adenosine.

h. Anagrelide, a PDE 3 inhibitor. Used in essential thrombocythemia and Polycythemia Vera.

i. Anti-Spasmodic as Dratuverine, inhibits PDE 4. Smooth muscle relaxant Used in irritable bowel syndrome, renal and biliary colics, dysmenorrhea and acceleration of labor.

\section{Conclusion}

Since the discovery of cAMP in 1957 by the late 1971 Noble Prize winner Sutherland the knowledge about the downstream effects of the cyclic nucleotide "second messenger" system has increased enormously. In particular the EPAC system has the potential for many yet unknown therapeutic applications. The discovery of the new cyclic-di-adenosine monophosphate system is another new promising challenge. Cell biology has done a great job in cyclic nucleotide research and clinical pharmacology will benefit without doubt.

\section{Acknowledgements}

None.

\section{Conflicts of interest}

The author declares that there are no conflicts of interest.

\section{Funding}

None.

\section{References}

1. Sutherland EW, Rall TW. The properties of an adenosine ribonucleotide produced with cellular particles ATP, $\mathrm{Mg}^{2+}$ and epinephrine or glucagon. J Am Chem Soc. 1957;79(13):3608

2. Cooper DMF. Bimodal regulation of adenylate cyclase. FEBS letters. 1982;138(2):157-163.

3. Cohen P. The role of protein phosphorylation in neural and hormonal control of cellular activity. Nature. 1982;296:613-619.

4. Strada SJ, Martin MW, Thompson MJ. General properties of multiple molecular forms of cyclic nucleotide phosphodiesterase in the nervous system. Adv Cycl Nucl Res. 1984;16:13-30.

5. Berridge MJ. Inositol triphosphate and diacylglycerol as second messengers. Biochem J. 1984;220(2):345-359.

6. Cohen P. The role of protein phosphorylation in hormonal control of enzyme activity. Eur J Biochem. 1985;154(3):439-448.

7. Hoeffler JP, Meyer TE, Yun Y. Cyclic AMP-responsive DNA binding protein: structure based on cloned placental cDNA. Science. 1988;242(4884):1430-1433.

8. Chen $\mathrm{G}$, Zou X, Watanabe $\mathrm{H}$, et al. CREB binding protein is required for both short-term and long-term memory formation. $J$ Neurosci. 2010;30(39):13066-13077.

9. Bodor J. Editorial: The Molecular Mechanisms of Cyclic AMP in Regulation of Immunity and Tolerance. Front. Immunol. 2017;8:76.

10. Cherfils J, Chardin P. GEFs: structural basis for their activation of small GTP-binding proteins. Trends Biochem Sci. 1999;24(8):306-311. 
11. Elliot MR, Ravichandran KS. The Dynamics of Apoptotic Cell Clearance. Dev Cell. 2016;38(2):1471-1460.

12. Grandoch M, Roscioni SS, Schmidt M. The role of Epac proteins, novel cAMP mediators in the regulation of immune, lung and neuronal function. Br J Pharmacol. 2010;159(2):265-284.

13. Lorenowicz MJ, van Gils J, de Boer M, et al. EPAC1-RAP1 signalling regulates monocyte adhesion and chemotaxis. $J$ Leukoc Biol. 2006;80(6):1542-1552.

14. Kammer GM. The adenylate cyclase-camp-protein kinase A pathway and regulation of the immune response. Immunol Today. 1988;9(7-8):222-229.

15. Klein U, Tu Y, Stolovitzky GA, et al. Gene expression profiling of B cell chronic lymphocytic leukemia reveals a homogenous phenotype related to memory B cells. J Exp Med. 2001;194(11):1625-1628.

16. Schaafsma D, Roscioni SS, Meurs H, et al. Monomeric G-proteins as signal transducers in airway physiology and pathophysiology. Cell Signal. 2008;20(10):1705-17014.

17. Murray AJ, Shewan DA. EPAC mediates cyclic AMP-dependent axon growth,guidance and regeneration. Mol Cell Neurosci. 2008;38(4):578-588.

18. Yang Z, Kirton HM, Al-Owais M, et al. EPAC2-RAP1 signalling regulates reactive oxygen species production and susceptibility to cardiac arrythmias. Antioxidants Redox Signaling. 2017;27(3):117-132.

19. Dzhura I, Chepurny OG, Leech CA, et al. Phospholipase C links EPAC2 activation to the potentation of glucose stimulated insulin secretion from mouse islet cells of Langerhans. J Islets. 2011;3(3):121-128.

20. Gelinas JN, Banko JL, Peters MM, et al. Activation of exchange protein activated by cyclic- AMP enhances long-lasting potentiation in the hypocampus. Learn Mem. 2008;15(6):403-411.

21. Ster J, de Bock F, Bertaso F, et al. EPAC mediates PACAP dependent long-term depression in the hippocampus. $J$ Physiol. 2009;587(1):101-113.

22. 22Parnell E, Palmar T.M, Yarwood S.J. (2015) The future of EPACtargeted therapies: agonism versus antagonism. Trends Pharmacol Sci 36(4): 203-214.

23. Kawamatawong T. Role of roflumilast, a selective phosphodiesterase inhibitor in airway disease. J Thorc Dis. 2017;9(4):1144-1154.

24. Lima KM, Vago JP, Caux TR, et al. The resolution of acute inflammation induced by cyclic AMP is dependent on annexin A. J Biol Chem. 2017;292(33):1358-1373.
25. Levy J, Zhoy DM, Zippin JH. Cyclic AMP Signalling in Inflammatory Skin. J Clin Exp Dermatol Res. 2016;7:326.

26. Kumar N, Goldminz AM, Kim N, et al. Phosphodiesterase 4-targeted treatment in autoimmune diseases. BMC Med. 2013;11:96.

27. Bai Y, Yang J, Eisele LE, et al. Two DHH subfamily 1 proteins in Streptococcus pneumoniae possess cyclic-di-AMP-phosphodieserase activity and affect bacterial growth and virulence. $J$ Bacteriol. 2013;195(22):5123-5132.

28. Sureka K, Choi PH, Precit M, et al. The cyclic dinucleotide c-diAMP is an allosteric regulator of metabolic enzyme function. Cell. 2016;158(6):1389-1401.

29. Kerkela R, Ulvila J, Magga J. Natriuretic Peptides in the Regulation of Cardiovascular Physiology and Metabolic Events. JAHA. 2015;4(10):e002423.

30. Zhao Y, Vanhoutte PM, Leung SW. Vascular nitric oxide: Beyond eNOS. J Pharmacol Sci. 2015;129(2):83-94.

31. Shabeeh H, Khan S, Jiang B, et al. Blood Pressure in Healthy Humans is Regulated by Neuronal NO Synthase. Hypertension. 2017;69(5):970-978.

32. Bachmann M, Waibler Z, Pleli T, et al. Type 1 Interferon Supports Nitric Oxide Synthetase in Murine Hepatoma Cells and Hepatocytes and during Experimental Acetaminophen-Induced Liver Damage. Front Immunol. 2017;8:890.

33. Camara AKS, Zhou Y, Wen PC, et al. Mitochondrial VDAC1; A Key Gatekeeper as Potential Therapeutic Target. Front Physiol. 2017;8:460.

34. Rao GH. Role of cyclic AMP and cyclic GMP as modulators of platelet cytosolic calcium. J Clin Prevent Cardiol. 2016;5(3):99-103.

35. Francis SH, Busch J, Corbin JD. cGMP-Dependent Protein Kinases and cGMP Phosphodiesterases in Nitric Oxide and cGMP Action. Pharmacol Rev. 2010;62(3):525-563.

36. Naafs MA, Fischer HR, van der Velden PC, et al. End-organ resistance to PTH infusion in hypercalcaemic and normocalcaemic patients with solid tumors. Acta Endocrinol (Copenh). 1986;113(4):543-550.

37. Naafs MAB, Fischer HRA, van der Velden PC. Changes in nephrogenous cAMP excretion and plasma cyclic AMP following treatment of hyperthyroidism. Acta Endocrinol (Copenh). 1984;10:477-481.

38. Naafs Michael AB. Pharmacodynamic Evaluation: Endocrinology. In: Hock FJ \& Gralinski MR, editors. Drug Discovery and Evaluation: Methods in Clinical Pharmacology. $2^{\text {nd }}$ edn, Springer Verlag Berlin, Heidelberg, New York, USA. 2017. 